\title{
Spintronic materials for hydrogen sensing
}

\author{
$\underline{\text { Wen-Chin Lin }}^{1^{*}}$, Chuan-Che Hsu ${ }^{1}$, Po-Chun Chang ${ }^{1}$, Yi-Hua Chen ${ }^{1}$, Chak-Ming Liu ${ }^{1}$, Chun-Te Wu ${ }^{2}$, \\ Hung-Wei Yen ${ }^{2}$ \\ ${ }^{1}$ Department of Physics, National Taiwan Normal University, Taipei 11677, Taiwan \\ ${ }^{2}$ Department of Materials Science and Engineering, Taipei 10617, Taiwan \\ *Corresponding author: wclin@ntnu.edu.tw
}

\begin{abstract}
$[\mathrm{Pd} / \mathrm{Fe}]_{2}$ multilayers were deposited on a flat $\mathrm{MgO}(001)$ substrate to study the effect of hydrogen on magnetic interlayer coupling. In an $\mathrm{Fe} / \mathrm{Pd} / \mathrm{Fe}$ interlayer coupled system, complex magnetic hysteresis behavior, including single, double, and triple loops, were measured as a function of the azimuthal angle $(\varphi)$ in a longitudinal and transverse direction. It was hypothesized that with a combination of a 2fold magnetic anisotropy energy in the bottom-Fe and a 4-fold MAE in the top-Fe, the complex magneto-optical Kerr effect hysteresis behavior could be clearly explained. Two well-split hysteresis loops with Kerr remanence of almost zero were measured by choosing a suitable Pd thickness and applying the magnetic field perpendicular to the easy axis of the bottom-Fe. The split double loops originated from the $90^{\circ}$-rotation of the top-Fe moment. On exposure to a hydrogen gas atmosphere, the separation of the two minor loops increased, indicating that Pd-hydride formation enhanced the ferromagnetic coupling between the two Fe layers. On the basis of these observations, we proposed that, by applying a suitable constant magnetic field, the top-Fe moment could undergo reversible $90^{\circ}$ rotation following hydrogen exposure. The results suggest that the Pd space layer used for mediating the magnetic interlayer coupling is sensitive to hydrogen, and therefore, the $[\mathrm{Fe} / \mathrm{Pd}]_{\mathrm{n}}$ multilayer system can function as a giant magnetoresistance-type sensor suitable for hydrogen gas.
\end{abstract}

Key words: Hydrogen sensor, Spintronic material, Magnetic thin film

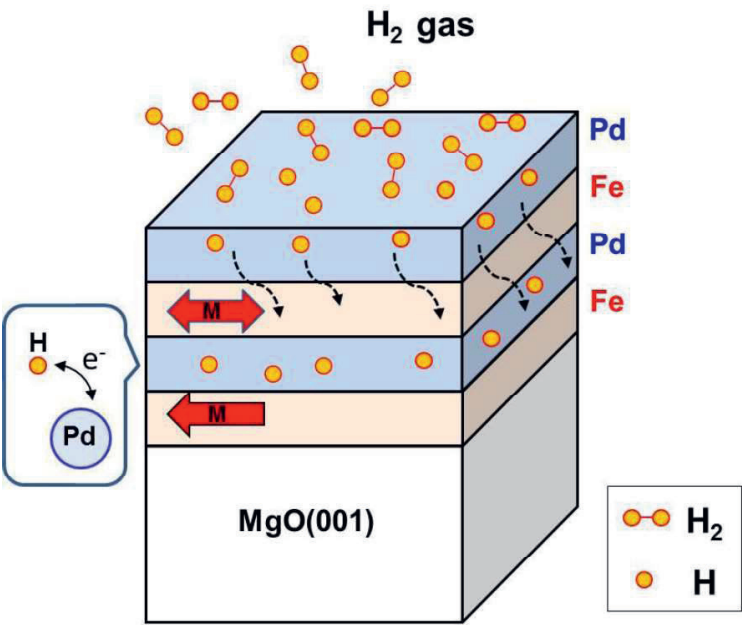

Fig. 1. Schematic of the effect of hydrogenation on the Pd-covered $\mathrm{Fe} / \mathrm{Pd} / \mathrm{Fe}$ trilayers on a $\mathrm{MgO}(001)$ substrate. Hydrogen molecules dissociated on the top Pd layer and diffused into the underlayers. The electronic structure of the Pd-mediate layer, and the interlayer magnetic ordering between the two $\mathrm{Fe}$ layers, is expected to be modulated through $\mathrm{Pd}$ hydride formation.
Pd has long been used as a high-efficient catalyst for the dissociation of hydrogen molecules into individual atoms. The effects of $\mathrm{Pd}$-assisted hydrogenation on magnetic properties have been reported in $\mathrm{Pd} / \mathrm{Fe}, \mathrm{Co}, \mathrm{Ni}$ bilayers, $[\mathrm{Pd} / \mathrm{Co}]_{n}$ multilayers, and Co-Pd alloys. Furthermore, hydrogen absorption and desorption can induce reversible changes in magnetic coercivity, remanence/saturation ratio, magnetic moment, magnetic anisotropy energy, and microscopic domain wall motion. These observations demonstrate the potential for using spintronic devices as hydrogen gas sensors. Magnetic interlayer exchange coupling, also known as Ruderman-Kittel-KasuyaYosida (RKKY) coupling, has been widely studied through the application of giant magnetoresistance (GMR) in magnetic storage and magnetic field sensors. Pd-related materials have been thoroughly studied and suggested as candidates for hydrogen sensors and hydrogen storage; however, very few studies have investigated Pd-mediated RKKY interlayer exchange coupling and the effect of 
hydrogen on the Pd-mediated interlayer coupling thus far. In the present study, as illustrated in Fig. 1, we examined the magnetic behavior of $\mathrm{Fe} / \mathrm{Pd} / \mathrm{Fe}$ trilayers on $\mathrm{MgO}(001)$ and the effect of hydrogenation on magnetic interlayer coupling between the two Fe layers.

a

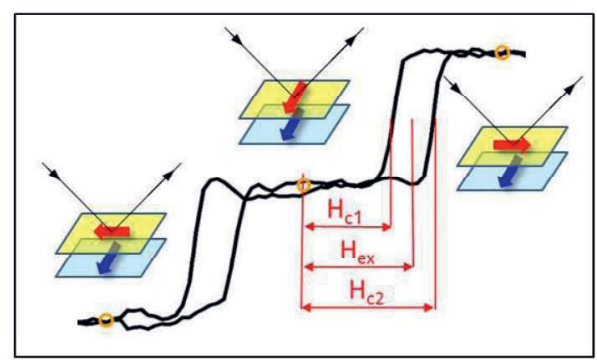

b

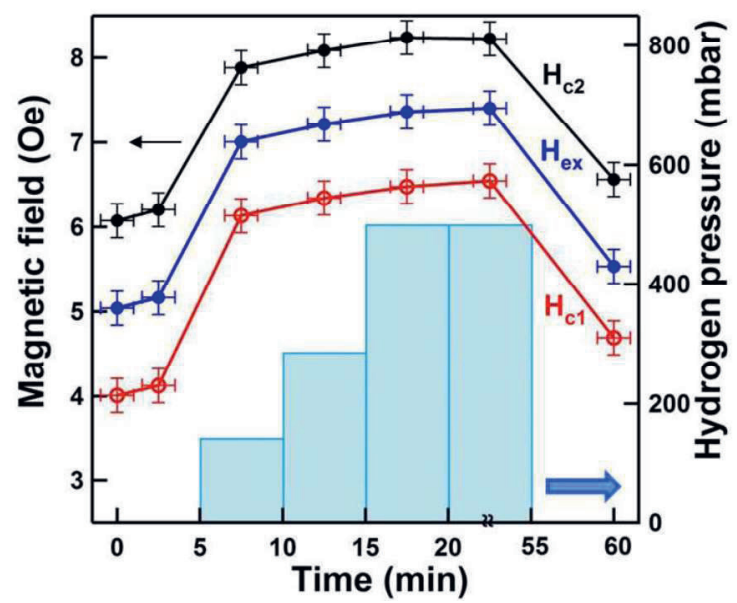

Fig. 2. (a) Schematic of the three types of magnetization ordering of $\mathrm{Fe} / \mathrm{Pd} / \mathrm{Fe}$ in the double loop. (b) Left: Magnetic field of $H_{c 1}, H_{c 2}$ and $H_{e x}$. Right: Corresponding $\mathrm{H}_{2}$ gas pressure in the measurement environment.

The characterization of a crystalline structure using high-resolution TEM confirmed the coherent growth of $\mathrm{Pd} / \mathrm{Fe}$ on $\mathrm{MgO}(001)$. For single-layer $\mathrm{Fe}$ on $\mathrm{Mg}(001)$, a uniaxial MAE was observed, which is attributable to the possible miscut of the substrate. In the $\mathrm{Fe} / \mathrm{Pd} / \mathrm{Fe}$ interlayer coupled system, complex magnetic hysteresis behavior, including single, double, and triple loops, was measured as a function of the $\varphi$ in a longitudinal and transverse direction. The bottom-Fe layer was affected by the miscut of the substrate and therefore a uniaxial (2-fold) MAE dominated the magnetic behavior. The top-Fe layer was not affected by the substrate considerably and its intrinsic crystalline (fourfold) MAE was more likely to dominate. Following the hypothesized combination of 2and 4-fold MAE in the bottom- and top-Fe layers, respectively, the complex MOKE hysteresis loops can be clearly explained. By selecting a suitable Pd thickness and applying the magnetic field perpendicular to the easy axis of the bottom-Fe layer, two well-split hysteresis loops with almost zero Kerr remanence were measured. These split double loops originated from the $90^{\circ}$ rotation of the topFe moment. Through exposure to a hydrogen gas atmosphere, separation of the two minor loops increased owing to the Pd-hydride formed in the space layer. The reversibility of this hydrogenation effect was then demonstrated. The increased switching field indicated that Pdhydride formation enhanced ferromagnetic interlayer coupling between the top- and bottom-Fe layers. Thus, we can conclude that when a suitable constant magnetic field is applied, the top-Fe moment will undergo a reversible $90^{\circ}$ rotation following hydrogen exposure. These results suggest that the $\mathrm{Pd}$ space layer, which mediates the magnetic interlayer coupling, is sensitive to the hydrogen atmosphere; the $[\mathrm{Fe} / \mathrm{Pd}]_{\mathrm{n}}$ multilayer system can therefore function as a GMR-type sensor suitable for hydrogen sensing.

\section{References}

[1] C.-C. Hsu, P.-C. Chang, Y.-H. Chen, C.-M. Liu, C.-T. Wu, W.-C. Lin, Reversible 90-Degree Rotation of Fe Magnetic Moment Using Hydrogen, Scientific Reports 8:3251 (2018); doi: 10.1038/541598-018-21712-3

[2] W.-C. Lin, C.-J. Tsai, H.-Y. Huang, B.-Y. Wang, V. R. Mudinepalli, H.-C. Chiu, Hydrogen-mediated Long-range Magnetic Ording in Pd-rich Alloy Film, Applied Physics Letters 106, 12404 (2015); doi: 10.1063/1.4905463

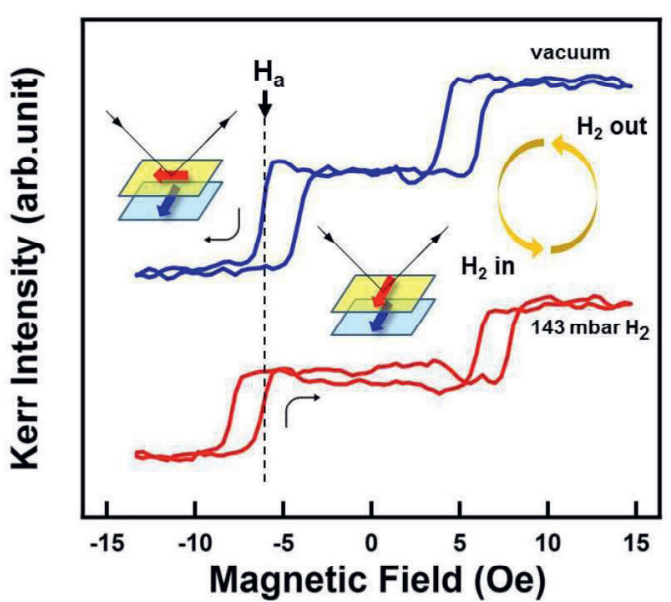

Fig. 3. Reversible $90^{\circ}$ rotation of the magnetic moment in the top-Fe layer through hydrogen charging. Following $\mathrm{H} 2$ gas absorption or desorption, the magnetization minor hysteresis loop shifted toward a larger or smaller coercivity field. When a suitable constant field $\mathrm{Ha}$ (as indicated by the dashed line) was applied, the hydrogen charge or discharge reverses the top-Fe layer by $90^{\circ}$. 